\title{
Approach to equilibrium in adiabatically evolving potentials
}

\author{
H. S. Samanta and J. K. Bhattacharjed \\ Department of Theoretical Physics, \\ Indian Association for the Cultivation of Science \\ Jadavpur, Calcutta 700 032, India \\ R.Ramaswamy壮 \\ School of Physical Sciences, Jawaharlal Nehru University, New Delhi 110 067, India
}

(Dated: February 8, 2020)

\begin{abstract}
For a potential function (in one-dimension) which evolves from a specified initial form, $V_{i}(x)$, to a different $V_{f}(x)$ asymptotically, we study the evolution, in an overdamped dynamics, of an initial probability density to its final equilibrium. There can be unexpected effects that can arise from the time dependence. We choose a time variation of the form $V(x, t)=V_{f}(x)+\left(V_{i}-V_{f}\right) e^{-\lambda t}$. For a $V_{f}(x)$, which is double welled and a $V_{i}(x)$ which is simple harmonic, we show that in particular, if the evolution is adiabatic, this results in a decrease in the Kramers' time characteristic of $V_{f}(x)$. Thus the time dependence makes diffusion over a barrier more efficient. There can also be interesting resonance effects when $V_{i}(x)$ and $V_{f}(x)$ are two harmonic potentials displaced with respect to each other that arise from the coincidence of the intrinsic time-scale characterizing the potential variation and the Kramers' time. Both these features are illustrated through representative examples.
\end{abstract}

PACS numbers: 05.10Gg

\section{INTRODUCTION}

The pioneering work of Kramers [1] on thermally activated barrier crossing has provided an understanding of the microscopic mechanism underlying the Arrhenius temperature dependence of crossing rates 2]. Several variants of the basic problem have subsequently been studied in the literature. A fair amount of attention has recently been devoted to the study of more complex nonequilibrium systems. These include the cases of diffusion over a barrier in the presence of harmonic force [3, 4, 5, 6, 7, 8, 9, 10] and diffusion over a fluctuating barrier 11, 12, 13, 14, 15, 16, 17]. The hallmark of the former situation is the phenomenon of stochastic resonance, where the signal-to-noise ratio of the system response to an applied harmonic force displays a local maximum as a function of the diffusion constant or the temperature. In fluctuating barriers, the discovery [12] that the mean first passage time has a minimum as a function of the correlation time characterizing the fluctuation has prompted a wide variety of investigations. The problem of surmounting potential barriers [17, 18, 19] has gained importance in other fields of science such as evolutionary computations 20, 21] and global optimization 22] as well.

In the present work we consider the situation of barrier crossing of a time-dependent potential which adiabatically evolves from $V_{i}(x)$ at $t=0$ to the potential $V_{f}(x)$ as $t \rightarrow \infty$. In such a situation there will be an eventual equilibrium distribution given by

$$
P_{e q} \sim \exp -\frac{V_{f}(x)}{\epsilon},
$$

where $\epsilon$ is the diffusion constant and the approach to this equilibrium will be governed by a characteristic time, which differs from the corresponding characteristic time for the stationary potential $V(x)=V_{f}(x)$. We note that the characteristic time is the same as Kramers' time where the potential $V_{f}(x)$ is one with a barrier .

Our main results are given in the next Section, where we derive the time-dependent probability distribution for a specific form of the time variation leading from the initial potential $V_{i}(x)$ to the final $V_{f}(x)$. Other forms of the time variation can be treated by a simple extension of the techniques outlined there. In Section III, two specific examples of evolving potentials are considered. When the time-scale of the perturbation matches the Kramers time for the

\footnotetext{
*Electronic address: tphss@mahendra.iacs.res.in

$\dagger^{\dagger}$ Electronic address: tpjkb@mahendra.iacs.res.in

‡Electronic address: r.ramaswamy@mail.jnu.ac.in
} 
stationary potential $V_{f}(x)$, there is a resonance which delays the onset of equilibrium. This case is treated within the time-dependent perturbation theoretic method of Dirac. The second case we study is one where $V_{i}(x)$ has a single minimum while $V_{f}(x)$ is bistable. By reducing to an effective two-state dynamics, we show that the Kramers time for the stationary potential $V_{f}(x)$ is reduced. The paper concludes with a summary and discussion in Section IV. Our result also sheds some light on the global optimization scheme recently introduced by Hunjan, Sarkar and Ramaswamy (HSR) [21, 31].

\section{THE TIME-DEPENDENT DISTRIBUTION}

For concreteness, we consider the time-dependent potential

$$
V(x, t)=V_{f}(x)+\left[V_{i}(x)-V_{f}(x)\right] e^{-\lambda t}
$$

which evolves via homotopy from $V_{i}(x)$ at $t=0$ to $V_{f}(x)$ at $t \rightarrow \infty$. The Fokker-Planck equation for the probability distribution $P(x, t)$ is

$$
\frac{\partial P}{\partial t}=\frac{\partial}{\partial x}\left(P \frac{\partial V}{\partial x}\right)+\epsilon \frac{\partial^{2} P}{\partial x^{2}},
$$

which, with the substitution [23, 24, 25],

$$
P(x, t)=\phi(x, t) \exp -\frac{V(x, t)}{2 \epsilon}
$$

reduces to

$$
\frac{\partial \phi}{\partial t}=H_{0} \phi+H_{1}(t) e^{-\lambda t} \phi
$$

where (primes denoting differentiation with respect to $x$ ),

$$
\begin{aligned}
H_{0} & =\epsilon \frac{\partial^{2}}{\partial x^{2}}+\frac{1}{2} V_{f}^{\prime \prime}-\frac{1}{4 \epsilon} V_{f}^{\prime 2} \\
H_{1} & =\left[\frac{\Delta V^{\prime \prime}}{2}-\frac{\lambda}{2 \epsilon} \Delta V-V_{f}^{\prime} \frac{\Delta V^{\prime}}{2 \epsilon}\right]-\frac{\left(\Delta V^{\prime}\right)^{2}}{4 \epsilon} e^{-\lambda t} \\
\text { and } & \\
\Delta V & =V_{i}(x)-V_{f}(x) .
\end{aligned}
$$

$H_{0}$ satisfies the eigenvalue equation

$$
H_{0} \psi_{n}=-E_{n} \psi_{n}(x)
$$

with nonpositive eigenvalues. By construction, the ground state has eigenvalue $E_{0}=0$, with the eigenfunction $\psi_{0}(x)=A \exp -V_{f}(x) / 2 \epsilon, A$ being a normalization constant. Denoting the space-independent part of $H_{1}$ by $V_{0}(t)$, the solution of Eq. (3) can be written as

$$
\phi(x, t)=\sum c_{n}(t) e^{-E_{n}(t)+\int V_{0}\left(t^{\prime}\right) d t^{\prime}} \psi_{n}(x) .
$$

Application of the standard techniques of the Dirac time-dependent perturbation theory leads to

$$
\dot{c}_{n}(t)=\sum_{m} c_{m}(t)\left\langle m\left|H_{2}\right| n\right\rangle e^{-\left(E_{m}-E_{n}\right) t}
$$


where

$$
H_{2}=e^{-\lambda t}\left(H_{1}-V_{0}\right)
$$

The perturbative expansion for the coefficients in Eq. (10) is in powers of $H_{2}$

$$
c_{n}(t)=\sum_{j=0}^{\infty} c_{n j}(t)
$$

with

$$
\dot{c}_{n 0}=0
$$

and for $j \geq 1$,

$$
\dot{c}_{n j}(t)=\sum_{m} c_{m, j-1}(t)\left\langle m\left|H_{2}\right| n\right\rangle e^{-\left(E_{m}-E_{n}\right) t} .
$$

It can be seen immediately that the $c_{n 0}$ 's are constants determined by the state of the system at $t=0$. Assuming that the system is in the equilibrium state of the potential $V_{i}(x)$ at $t=0$, namely

$$
P(x, 0)=A_{0} \exp -\frac{V_{i}}{\epsilon}
$$

this implies that

$$
\phi(x, 0)=P(x, 0) \exp \frac{V_{i}}{2 \epsilon}=A_{0} \exp -\frac{V_{i}}{2 \epsilon}
$$

with normalization constant $A_{0}$. The constants $c_{n 0}$ are now determined from the intial condition, as

$$
c_{n 0}=A_{0} \int_{-\infty}^{\infty} d x \psi_{n}(x) \exp -\frac{V_{i}}{2 \epsilon}
$$

Substituting this in Eq. (15), the complete solution to the problem can be obtained using Eqs. (13) and (10).

Note that as $t \rightarrow \infty, \phi(x, t)$ in Eq. (10) tends to $c_{00} \psi_{0}(x)=c_{00} \exp -\frac{V_{f}}{2 \epsilon}$, and therefore,

$$
P(x, t \rightarrow \infty) \rightarrow A \exp -\frac{V_{f}}{\epsilon},
$$

the equilibrium distribution corresponding to $V_{f}(x)$.

Also note from Eqs. (1112) that since $H_{2}$ has the time dependence exp $-\lambda t$, there will be a resonance when $E_{m}-E_{n}=\lambda$, giving a secular growth of the first order term, $c_{n 1}(t) \propto t$. This is analogus to case of the timedependent perturbation theory [26, 27] in quantum mechanics.

\section{APPLICATIONS}

\section{A. Case I}

Consider first a case where the initial and final potential have the same number of minima. Specifically, we take $V_{i}=(x-a)^{2}$ and $V_{f}(x)=x^{2}$, namely harmonic potentials that are spatially displaced. This leads to

$$
\Delta V=a^{2}-2 a x,
$$




$$
H_{1}=\left[-\frac{\lambda\left(a^{2}-2 a x\right)}{2 \epsilon}+\frac{2 a x}{\epsilon}\right]-\frac{a^{2} e^{-\lambda t}}{\epsilon},
$$

which has the space-independent part

$$
V_{0}(t)=-\frac{\lambda a^{2}}{2 \epsilon}-\frac{a^{2} e^{-\lambda t}}{\epsilon},
$$

giving

$$
H_{2}(x, t)=\frac{a x}{\epsilon}(\lambda+2) e^{-\lambda t}
$$

The leading term in the expansion, namely

$$
H_{0}=\epsilon \frac{\partial^{2}}{\partial x^{2}}+1-\frac{x^{2}}{\epsilon}
$$

has the eigenvalue spectrum $E_{n}=2 n(n=0,1,2, \ldots)$ with eigenfunctions

$$
\psi_{n}(x)=\left[2^{n} n ! \sqrt{\epsilon \pi}\right]^{-1 / 2} \mathcal{H}_{n}(x / \sqrt{\epsilon}) \exp -\frac{x^{2}}{2 \epsilon}
$$

where $\mathcal{H}_{n}(y)$ are the usual Hermite polynomials. The time-dependent probability is therefore

$$
P(x, t)=\phi(x, t) \exp \left(-\frac{x^{2}}{2 \epsilon}-\frac{\left(a^{2}-2 a x\right)}{2 \epsilon} e^{-\lambda t}\right)
$$

giving, for $\phi(x, t)$, the expansion (cf. Eq. (10))

$$
\phi(x, t)=\sum_{n} c_{n}(t) \exp \left(-2 n t-\frac{a^{2}}{2 \epsilon}\left(1-e^{-\lambda t}\right)\right) \psi_{n}(x) .
$$

To first-order in the perturbation expansion, we find

$$
c_{n}(t=0)=\left[\frac{a}{2 \epsilon^{\frac{1}{2}}}\right]^{n} \frac{e^{-\frac{a^{2}}{2 \epsilon}}}{\left[2^{n} n !\right]^{\frac{1}{2}}} .
$$

At the lowest order of perturbation theory, only the $c_{n 0}$ 's, which are given by Eq. (28) matter. Straightforward algebra now shows that

$$
\begin{aligned}
P(x, t)= & {\left[\frac{1}{\pi \epsilon}\right]^{\frac{1}{2}} \exp -\frac{x^{2}+a^{2}}{\epsilon}+\frac{2 a x e^{-2 t}}{\epsilon} } \\
& \exp \frac{a x\left(e^{-\lambda t}\right)-e^{-2 t}}{\epsilon}+O\left(c_{n 1}\right) .
\end{aligned}
$$

Since all terms to first order have not been included in the perturbation, the equilibrium distribution is not properly normalized as $t \rightarrow \infty$ and has the extraneous factor $\exp \left(-a^{2} / \epsilon\right)$. After computation of $c_{n 1}$ from Eq. (15) we find, after taking the appropriate matrix elements and carrying out the integration over time, that 


$$
\begin{aligned}
c_{n 1}(t) & =\frac{a}{\epsilon}\left(\frac{n+1}{2}\right)^{\frac{1}{2}}\left[1-e^{-\lambda t}\right] c_{n+1}(0) \\
& +\left(\frac{\lambda+2}{\lambda-2}\right) \frac{a}{\epsilon}\left(\frac{n}{2}\right)^{\frac{1}{2}}\left[1-e^{-(\lambda-2) t}\right] c_{n-1}(0)
\end{aligned}
$$

The coefficients $c_{n \pm 1}(0)$ are known from Eq. (28) and after some amount of algebra we get, correct to first order in the perturbing 'Hamiltonian',

$$
P(x, t)=\left(\frac{1}{\pi \epsilon}\right)^{\frac{1}{2}} e^{-x^{2} / \epsilon}\left[1+\frac{2 a x e^{-\lambda t}}{\epsilon}+\frac{4 a x\left(e^{-2 t}-e^{-\lambda t}\right)}{(\lambda-2) \epsilon}+\ldots\right]
$$

The effect of time-dependence in the potential can be seen by contrasting the above result, Eq. (31) with the sudden limit, when the potential is instantaneously changed from $V_{i}(x)$ to $V_{f}(x)$. The initial probability distribution corresponding to $V_{i}(x)$ is $\left(\frac{1}{\pi \epsilon}\right)^{\frac{1}{2}} e^{-\frac{(x-a)^{2}}{\epsilon}}$ and this approaches the equilibrium distribution corresponding to $V_{f}(x)$ as

$$
P(x, t)=\left(\frac{1}{\pi \epsilon}\right)^{\frac{1}{2}} e^{-\frac{x^{2}}{\epsilon}}\left[1+\frac{2 a x e^{-2 t}}{\epsilon}\right] .
$$

The coefficient $\frac{2 a x}{\epsilon}$ of $e^{-2 t}$ has the extra factor $(1+2 /(\lambda-2))$. Note that the time-dependent perturbation effectively keeps the system from attaining equilibrium by always managing to cause transition to neighbouring states. The approach to equilibrium depends on the value of the adiabaticity parameter, $\lambda$, and there are three different regimes of interest.

- When $\lambda>2$, the approach to equilibrium is governed by $e^{-2 t}$ but the coefficient of this term is significantly increased.

- If $\lambda<2$, the approach is controlled by $e^{-\lambda t}$ and in the long time limit

$$
P(x, t) \sim \frac{1}{(\pi \epsilon)^{1 / 2}} e^{-x^{2} / \epsilon}\left[1+\left(\frac{4 a x / \epsilon}{2-\lambda}\right) e^{-\lambda t}\right] .
$$

- Finally, we have the extremely interesting situation of $\lambda \simeq 2$, in which case

$$
P(x, t)=\frac{1}{(\pi \epsilon)^{1 / 2}} e^{-\frac{x^{2}}{\epsilon}}\left(1+\frac{4 a x t}{\epsilon} e^{-2 t}+\ldots\right)
$$

This is the resonance that we have discussed already, which shows up as the coefficient of the usual correction to $P_{e q}(x)$ diverging with time.

This divergence of the coffecient of $e^{-2 t}$ in Eq.(34) would eventually get transferred to the argument of the exponential function as is usual in such cases. This can be explicitly verified in this case, because an exact solution for harmonic potentials has been written down by Hänggi and Thomas [28]. The answer for $P(x, t)$, adapting the work of Hänggi and Thomas to this situation is

$$
P(x, t)=\left[\frac{1}{\pi \epsilon\left(1-e^{-4 t}\right)}\right]^{1 / 2} \exp \left[-\frac{\left(x-a e^{-2 t}\left[1+2\left(1-e^{-(\lambda-2) t}\right) /(\lambda-2)\right]\right)^{2}}{\epsilon\left(1-e^{-4 t}\right)}\right]
$$

If we expand the exponential in powers of ' $a{ }^{\prime}$ all the three cases cited above are exactly reproduced. This shows that the method of quantum mechanical time dependent perturbation theory that we have adopted here in capable of yielding the correct results. 


\section{B. Case II}

We now turn to a situation where an initially single well structure, $V_{i}=x^{2}$, crosses over to a double well structure, $V_{f}=-\frac{x^{2}}{2}+\frac{x^{4}}{4}$, as $t \rightarrow \infty$. The approach to equilibrium in double well potential is governed by the Kramers time, the long time scale coming from the possibility of noise induced hopping. Following the procedure outlined in Eqs. (4 5), we get

$$
\begin{aligned}
H_{0} & =-\epsilon \frac{d^{2}}{d x^{2}}+\frac{\left(x^{3}-x\right)^{2}}{4 \epsilon}-\frac{1+3 x^{2}}{2} \\
H_{1} & =\frac{3}{2}\left(1-x^{2}\right)-\frac{1}{4 \epsilon} x^{2}\left(3-x^{2}\right)\left(1-x^{2}\right) \\
& -\frac{\lambda}{8 \epsilon} x^{2}\left(6-x^{2}\right)+\frac{1}{4 \epsilon} x^{2}\left(3-x^{2}\right)^{2}\left(1-e^{-\lambda t}\right) .
\end{aligned}
$$

The low-lying part of the eigenvalue spectrum of $H_{0}$ is characterized by a set of close doublets with exponentially small separations, while the gap between two doublets is of $O(1)$. The ground state $E_{0}=0$, while the first excited state is the ground state of the supersymmetric partner of

$$
\frac{\left(V_{f}^{\prime}\right)^{2}}{4 \epsilon}-\frac{V_{f}^{\prime \prime}}{2}
$$

and is exponentially small [29, 30]. The next excited state has eigenvalue approximately 2 , and hence we can treat the dynamics of the low lying states as that of a two level system. Denoting the two states by $\phi_{0}$ and $\phi_{1}$, with eigenvalues 0 and $\delta$, then

$$
\phi(x, t)=c_{0}(t) \phi_{0}(x)+c_{1}(t) e^{-\delta t} \phi_{1}(x)
$$

The dynamics of $c_{0}$ and $c_{1}$ is governed by

$$
\begin{aligned}
& \dot{c}_{0}=\left\langle\phi_{0}\left|H_{1}\right| \phi_{0}\right\rangle e^{-\lambda t} c_{0}(t)+\left\langle\phi_{0}\left|H_{1}\right| \phi_{1}\right\rangle c_{1}(t) e^{-(\lambda+\delta) t} \\
& \dot{c}_{1}=\left\langle\phi_{1}\left|H_{1}\right| \phi_{0}\right\rangle e^{-(\lambda-\delta) t} c_{0}(t)+\left\langle\phi_{1}\left|H_{1}\right| \phi_{1}\right\rangle e^{-\lambda t} c_{1}(t)
\end{aligned}
$$

Since the perturbation $H_{1}$ is even, $\left\langle\phi_{1}\left|H_{1}\right| \phi_{0}\right\rangle=0$, decoupling $c_{0}$ and $c_{1}$ Integrating Eq. (41) and dropping terms like $e^{-2 \lambda t}$ which are unimportant for $t>\lambda^{-1}$, we find

$$
\dot{c}_{1}=c_{1}\left\langle\phi_{1}\left|\widetilde{H_{1}}\right| \phi_{1}\right\rangle e^{-\lambda t}
$$

where

$$
\widetilde{H_{1}}=\frac{3}{2}\left(1-x^{2}\right)+\frac{1}{2 \epsilon} x^{2}\left(3-x^{2}\right)\left(1-x^{2}\right)-\frac{\lambda}{8 \epsilon} x^{2}\left(6-x^{2}\right)
$$

The primary contribution to $\left\langle\phi_{1}\left|\widetilde{H_{1}}\right| \phi_{1}\right\rangle$ comes from the vicinity of $x=1$ since $\phi_{1}$ is an antisymmetric wave function strongly peaked near $x \pm 1$. Since $\left\langle\phi_{1}\left|\widetilde{H}_{1}\right| \phi_{1}\right\rangle \simeq-\frac{5 \lambda}{4 \epsilon}$, we have

$$
c_{1}(t)=B \frac{1-e^{-\lambda t}}{\lambda} \exp -\frac{5 \lambda}{4 \epsilon}
$$

with $B$ a constant of integration. From Eq. (39) we find, after a series of standard manipulations, that 


$$
\begin{aligned}
\phi(x, t) & \simeq N \phi_{0}(x)\left[1+\frac{\phi_{1}(x)}{\phi_{0}(x)} e^{-\delta t-\frac{3\left(1-e^{-\lambda t}\right) \alpha}{2 \lambda}}\right] \\
& \equiv N \phi_{0}(x)\left[1+f(x) e^{-\delta_{\text {eff }} t}\right] .
\end{aligned}
$$

In the above $\alpha$ is a measure of the strength of the ground state wave function at the origin, and where

$$
\delta_{\mathrm{eff}}=\delta+\frac{3\left(1-e^{-\lambda t}\right) \alpha}{2 \lambda t}
$$

The inverse of $\delta_{\text {eff }}$ gives the effective Kramers time for the system and is shorter than the scale for the time independent system. This speeding up is most effect in the adiabatic limit, namely for $\lambda \ll 1$.

\section{DISCUSSION AND SUMMARY}

The above result is a simple analogue of the global optimisation principle on an evolving energy landscape. In this case one is interested in finding the minima of a multidimensional potential energy surface which constitutes the energy landscape in problems such as protein folding or finding the ground state configuration of atomic or molecular clusters. The observation of HSR 21, 31], that continuously and adiabatically varying potentials assist approach to the desired configuration at $t \rightarrow \infty$ by avoiding trapping in local minima. We have shown in a model system, a similar time dependence, the decrease of Kramers time makes escape from a trapping potential easier.

The above demonstration of a reduced Kramers' time is for a one dimensional system. The extension to two dimensions is reasonably straightforward following the technique in ref.[30] when a well defined tunneling path exists between the two minima. Extensions to more general situations and to three dimensions is being investigated. We have also seen that this technique of dealing with time dependent perturbations can model the stochastic resonance as a kind of parametric resonance. With the emerging importance of stochastic resonance in biological systems [10] it is possible that yet another way of looking at stochastic resonance can yield new insights.

[1] H. A. Kramers, Physica (Utrecht) 7, 284 (1940).

[2] For a review, see P. Hänggi, P. Talkner and M. Borkovec, Rev. Mod. Phys. 62, 251 (1990).

[3] R. Benzi, A. Sutera and A. Vulpiani, J. Phys. A 14, L453 (1981).

[4] B. McNamara, K. Wiesenfeld, and R. Roy, Phys. Rev. Lett. 60, 2626 (1988).

[5] L. Gammaiton, F. Marchesoni, E. Menichella Saetta, and S. Santucci, Phys. Rev. Lett. 62, 349 (1989).

[6] D. L. Stein, R. G. Palmer, J. L. Van Hemmen, and C. R. Doering, Phys. Lett. A 136, 393 (1989).

[7] M. Büttiker and R. Landauer, Phys. Rev. Lett. 49, 1739 (1982).

[8] S.Upadhyay, J.Phys.A 24, L1293 (1991)

[9] L.Gammaitoni, P.Hänggi, P.Jung, F.Marchesoni, Rev.Mod.Phys. 70, 223 (1998)

[10] P.Hänggi, Chem.Phys.Chem. 3, 285 (2002)

[11] P.Hänggi, Phys.Lett.A 78, 304 (1980)

[12] C. R. Doering and J. C. Gadoua, Phys. Rev. Lett. 69, 2318 (1992).

[13] J. Maddox, Nature 359, 771(1992).

[14] U. Zürcher and C. R. Doering, Phys. Rev. E 47, 3862 (1993).

[15] C. Van den Broeck, Phys. Rev. E 47, 4579 (1993).

[16] P. Pechukas and P. Hänggi, Phys. Rev. Lett. 73, 2772 (1994).

[17] J. Lehmann, P. Reimann and P. Hänggi, Phys. Rev. Lett. 84, 1639 (2000).

[18] R.S.Maier, D.L.Stein, Phys.Rev.Lett. 86, 3942 (2001)

[19] J.Lehmann, P.Reimann and P.Hänggi, Phys.Stat.Sol.(b) 237, 53 (2003)

[20] J. Dunkel, W. Ebeling, L. Schimansky-Geier and P. Hänggi, Phys. Rev. E 67, 061118 (2003).

[21] J. S. Hunjan, S. Sarkar, and R. Ramaswamy, Phys. Rev. E 66, 046704 (2002).

[22] R.Horst and P.M.Pardalos, Handbook of Global Optimization (Kluwer Academic Press, Dordrecht, 1995) see also J.P.K.Doye, in Global Optimization-Selected Case Studies, edited by J.D.Printer (Kluwer Academic Press, Dordrecht, 2001) 
[23] H.Tomota, A.Ito, H.Kidachi, Prog.Theor.Phys. 56, 786 (1976)

[24] P.Hänggi and H.Thomas, Phys.Rep. 88, 207 (1982)

[25] H. Risken, The Fokker Planck Equation, (Springer-Verlag, Berlin, 1984)

[26] P. W. Laughoff, S. T. Epstein and M. Karplus, Rev. Mod. Phys. 44, 602 (1972).

[27] K. Bhattacharyya, Proc. Roy. Soc. Lond A 394, 345 (1984).

[28] P.Hänggi and H.Thomas, Z.Physik B, 22, 295 (1975)

[29] M. Bernstein and L. S. Brown, Phys. Rev. Lett. 92, 1933 (1984).

[30] J. K. Bhattacharjee and K. Banerjee, J. Phys. A 22, L1141 (1989).

[31] J. S. Hunjan, G. S. Matharoo, S. Sarkar, and R. Ramaswamy, to be published. 